\title{
LOCAL COHOMOLOGY OF MODULE OF DIFFERENTIALS OF INTEGRAL EXTENSIONS
}

\author{
S. P. DUTTA
}

\begin{abstract}
The main focus of this paper is on determining the highest non-vanishing local cohomology modules of $\Omega_{B / R}, \Omega_{B / V}$ $\left(\Omega_{B / k}\right)$ where $R$ is either a complete regular local ring or a complete local normal domain with coefficient ring $V$ (field $k$ ) and $B$ is its integral closure in an algebraic extension of $Q(R)$. Similar problem is also studied over a normal domain $R$ containing a field $\mathrm{k}$ of characteristic 0 . In this connection new observations on the direct summand property for integral extensions are also presented.
\end{abstract}

\section{INTRODUCTION}

In this note we mainly address the following problem: let $R$ be a normal domain containing a field $\mathrm{k}$ of characteristic 0 or let $(R, m)$ be a complete regular local ring/complete local normal domain of dimension $n$ with co-efficient ring $V$ (field $k$ ). Let $K$ denote the field of fractions of $R$ and let $B$ denote the integral closure of $R$ in an algebraic extension $F$ of $K$. Let $\Omega_{B / R}, \Omega_{B / V}\left(\Omega_{B / k}\right)$ denote the module of differentials of $B$ over $R, V(k)$ respectively.

Problem. Determine whether $\operatorname{Hom}_{R}\left(\Omega_{B / k}, R\right)\left(\operatorname{Hom}_{R}\left(\Omega_{B / V}, R\right)\right) \neq$ $\left.0, \operatorname{Hom}_{R}\left(\Omega_{B / R}, R\right)\right) \neq 0$ or determine the highest non-vanishing local cohomology modules with respect to $m$ of $\Omega_{B / V}\left(\Omega_{B / k}\right)$ and $\Omega_{B / R}$.

In section 2 we prove several lemmas that will be used in the proofs of theorems in section $3 \&$ section 4 . The corresponding results include providing sufficient conditions for a) extensions $A \rightarrow B$ so that $\Omega_{B / A}$ becomes $B$-flat, $\Gamma_{B / A}$ becomes null and for b) extensions $A \rightarrow B \rightarrow C$ so that the corresponding right exact sequence of module of differentials becomes left exact. Another useful tool in this respect is theorem 2.3 where by utilizing Popescu's work ([11], [14]) we provide a simple proof of the fact that for any geometrically regular extension of Noetherian rings $A \rightarrow B, \Omega_{B / A}$ is $B$-flat and $\Gamma_{B / A}=0$.

AMS Subject Classification: 13D02, 13D22, 13D45, 13N05

Key words and phrases: module of differentials, integral extension, geometric regularity, local cohomology, formally unramified extensions. 
The second part of this result is originally due to M. André ([1]) and later it was proved by Popescu ([11]) via his characterization of geometrically regular extensions. A stronger version of this result has been proved by Gabber \& Ramero (Cor. 5.6.25, [4]).

As a corollary to lemma 2.1 it has been pointed out that for any integral domain $A$ integral over a complete local normal domain $(R, m)$ of dimension $n$ such that $Q(A)$ is separably algebraic over $Q(R), \mathrm{H}_{m}^{n}\left(\Omega_{A / R}\right)$ $=0$.

One of our main results in section 3 is the following.

Theorem 3.5. Let $R$ be a normal domain of dimension $n$ containing a field $k$ of characteristic 0 with a non-null derivation $D \in \operatorname{Der}_{k}(R)$ and let $B$ be its integral closure in an algebraic extension $F$ of $K$. Then $\operatorname{Hom}_{R}\left(\Omega_{B / k}, R\right) \neq 0$. If $(R, m)$ is a complete local normal domain of dimension $n$ with maximal ideal $m$ and a non-null derivation $D \in$ $\operatorname{Der}_{k}(R)$, then $\mathrm{H}_{m}^{n}\left(\Omega_{B / k}\right) \neq 0$.

The two key steps in the proof of this theorem are Theorem 3.1 and Proposition 3.4 where the same statement is proved for a complete d.v.r. and for a d.v.r. with a non-null derivation respectively. In these proofs we provide a prescription for defining a non-null $R$-linear map: $\Omega_{B / k} \rightarrow R$ that depends on the non-null derivation $D$.

Next we deal with complete local domains. We would like to mention here that if $(C, q)$ is a complete local domain of dimension $n$ with coefficient ring $V(k)$, then $\Omega_{C / V}\left(\Omega_{C / k}\right)$ is not necessarily a finitely generated $C$-module. We prove the following:

Theorem 3.6. Let $(C, q)$ be a complete local domain of dimension $n \geq 2$ with coefficient ring $V$ (field $k$ ). Let $(R, m)$ be a power series ring over $V(k)$ contained in $C$ such that $C$ is a module finite extension of $R$. We have the following:

i) if $Q(C) / Q(R)$ is separably algebraic then $\mathrm{H}_{q}^{n}\left(\Omega_{C / V}\right)\left(\mathrm{H}_{q}^{n}\left(\Omega_{C / k}\right)\right) \neq 0$, ii) if characteristic of $k=p>0, k$ perfect and $\Omega_{Q(C) / k} \neq 0$, then $\mathrm{H}_{q}^{n}\left(\Omega_{C / k}\right) \neq 0$ and

iii) if $Q(C) / Q(R)$ is separably algebraic, then $\mathrm{H}_{q}^{n}\left(\Omega_{C / R}\right)=0$; moreover if $\Omega_{C / R} \neq 0$ and $C$ is normal, then $\mathrm{H}_{q}^{n-1}\left(\Omega_{C / R}\right) \neq 0$.

Theorem 3.7., the final result of this section, can be viewed as an extension of the theorem on purity of branch locus ([10], [15]) to infinitely generated integrally closed domains $B$ integral over a regular local ring $R$.

In section 4 we deal with the situation where $R$ is a complete regular local ring and $B$ is the absolute integral closure of $R$. Our problem is irrelevant in positive characteristic, since $\Omega_{B / k}=0$ and $\Omega_{B / R}=0$ i.e. 
$k \rightarrow B$ and $R \rightarrow B$ are unramified extensions. Due to theorem 3.5 we focus our attention on mixed characteristic. In this part of our work the highest non-vanishing local cohomology of $\Omega_{B / R}$ gets connected with the direct summand property for integral extensions of $R$ conjectured by M. Hochster ([7]). Hochster proved the equicharacteristic case of his conjecture in early seventies ([7]). In 2016 Y. André ([2]) proved this conjecture in mixed characteristic by using aspects of Almost Ring Theory introduced by Faltings in his work on $p$-adic Hodge theory. Afterwards Bhatt ([3]) gave a short proof of this conjecture and its derived variant. Both proofs use the notion of perfectoid geometry. The main theorem in section 4 is the following:

Theorem 4.1. Let $(R, m)$ be a complete regular local ring of dimension $\mathrm{n}$ with coefficient ring $V$ of mixed characteristic $p$ and let $B$ denote its absolute integral closure. We have the following:

i) $\Omega_{B / V}=p \Omega_{B / V}, \Omega_{B / R}=p \Omega_{B / R}$. These observations imply the following:

a) For any ideal $I$ of $R$ of the form $I=\left(p, x_{1}, . ., x_{i-1}\right)$ generated by a part of a system of parameters containing $p$ of $R, H_{I}^{i}\left(\Omega_{B / V}\right)=0$ and $H_{I}^{i}\left(\Omega_{B / R}\right)=0$. In particular $H_{m}^{n}\left(\Omega_{B / V}\right)=0$ and $H_{m}^{n}\left(\Omega_{B / R}\right)=0$.

b) $\widehat{\Omega}_{B / V}=0=\widehat{\Omega}_{B / R}$, where $(\widehat{-})$ denotes the I-adic completion. And hence $V \rightarrow B, R \rightarrow B, V \rightarrow \widehat{B}$ and $R \rightarrow \widehat{B}$ are all formally unramified extensions.

ii) Non-vanishing of $\mathrm{H}_{m}^{n-1}\left(\Omega_{B / R}\right)$ implies the direct summand property for integral extensions of $R$ (equivalently descent of flatness for integral extensions of Noetherian rings ([12])) and the converse is also true.

Non-vanishing of $\mathrm{H}_{m}^{n-1}\left(\Omega_{B / V}\right)$ implies the direct summand property for integral extensions over $R$.

For part i) we do not need $R$ to be regular. Actually we prove a much more general statement.

As a Corollary, due to Y. André's proof of the direct summand conjecture in mixed characteristic, it follows that $\mathrm{H}_{m}^{n-1}\left(\Omega_{B / R}\right) \neq 0$.

We do not know whether part ii) of the above theorem is valid in equicharacteristic zero.

As corollaries to Proposition 4.2 we derive the following over $R=$ $V\left[\left[X_{1}, \ldots, X_{n-1}\right]\right]$ and $m, B$ as above: i) for any $i>0$, if $H_{m}^{i+1}(B) \neq 0$, then $H_{m}^{i}\left(\Omega_{B / R}\right) \neq 0$ and ii) for any $i \geq 0$ there exists an exact sequence $0 \rightarrow \oplus_{1}^{n-1} \operatorname{Ext}_{R}^{n-i-1}(B, R) \rightarrow \operatorname{Ext}_{R}^{n-i}\left(\Omega_{B / R}, R\right) \rightarrow \operatorname{Ext}_{R}^{n-i}\left(\Omega_{B / V}, R\right) \rightarrow 0$ 
In particular

$$
0 \rightarrow \underset{1}{n-1} B^{*} \rightarrow \operatorname{Ext}_{R}^{1}\left(\Omega_{B / R}, R\right) \rightarrow \operatorname{Ext}_{R}^{1}\left(\Omega_{B / V}, R\right) \rightarrow 0
$$

is exact.

Our final theorem deals with local cohomology with respect to the ideal $\left(x_{1}, \ldots, x_{n-1}\right)$ generated by a part of a regular system of parameters. The second assertion in this theorem provides an apparently approachable sufficient condition for the direct summand property.

Theorem 4.3. Let $(R, m)$ be a complete regular local ring in mixed characteristics $p>0$ with co-efficient ring $V$, and $B$ be its absolute integral closure. Let $p, x_{1}, \ldots, x_{n-1}$ be a system of parameters of $R$ such that $x_{1}, \ldots, x_{n-1}$ form a regular system of parameters. Let $\underline{x}$ denote the ideal $\left(x_{1}, \ldots, x_{n-1}\right)$ in $R$. Then $\mathrm{H}_{\underline{x}}^{n-1}\left(\Omega_{B / V}\right) \neq 0$. Non-vanishing of $\mathrm{H}_{\underline{x}}^{n-1}\left(\Omega_{B / R}\right)$ implies the direct summand property for integral extensions of $R$.

Corollary. If $\mathrm{H}_{p}^{0}\left(\mathrm{H}_{\underline{x}}^{n-1}\left(\Omega_{B / V}\right)\right) \neq 0$, then $\mathrm{H}_{m}^{n-1}\left(\Omega_{B / V}\right) \neq 0$.

Let us note that some of the results proved in section 4 are not valid in equicharacteristic zero. I do not know answers to several questions that came up during the course of this work. A few of these questions related to this paper are mentioned at the end.

For definition of $\Gamma_{S / R}$ and for basic properties of $\Omega_{S / R}$ and $\Gamma_{S / R}$ the reader is referred to $([5],[14])$.

Notations. Given an extension $R \rightarrow S$ the corresponding module of differentials is denoted by $\Omega_{S / R}$ (instead of $\left.\Omega_{S / R}^{1}\right)$; d.v.r. stands for discrete valuation ring; for any integral domain (ring) $C, Q(C)$ denotes the field of fractions (quotient ring) of $C$; for any local ring $(R, m), E$ denotes the injective hull of $R / m$ over $R, E(k(P))$ denotes the injective hull of $R / P$ over $R$ and for any $R$-module $M, M^{\vee}$ stands $\operatorname{Hom}_{R}(M, E)$, $M^{*}$ stands for $\operatorname{Hom}_{R}(M, R)$.

\section{SECTION 2.}

In this section we are going to prove several lemmas and propositions that will be used in section 3 and section 4 .

2.1. Lemma. Let $R$ be a normal domain, $R \hookrightarrow A$ an integral extension such that $A$ is an integral domain and $Q(R) \hookrightarrow Q(A)$ is a separable algebraic extension. Then $\operatorname{Hom}_{R}\left(\Omega_{A / R}, R\right)=0$.

Proof. For any $a \in A, a \neq 0$, the minimal polynomial $f(X)$ for $a$ has all its co-efficient in $R$; separability of $Q(A)$ over $Q(R)$ implies $f^{\prime}(a) \neq 0$. Since $f(a)=0, f^{\prime}(a) d a=0 \ldots \ldots \ldots \ldots \ldots \ldots \ldots \ldots \ldots \ldots$ 
Since $f^{\prime}(a)$ is integral over $R$ and $A$ is an integral domain, an integral equation of smallest degree satisfied by $f^{\prime}(a)$ is of the form:

$$
\left[f^{\prime}(a)\right]^{t}+r_{1}\left[f^{\prime}(a)\right]^{t-1}+\cdots+r_{t}=0, r_{i} \in R
$$

where $r_{t} \neq 0$. This implies, due to $(1)$, that $r_{t} d a=0$. This is true for every $a$ in $A-R$. Thus $\Omega_{A / R}$ is a torsion module over both $A$ and $R$ and hence $\operatorname{Hom}_{R}\left(\Omega_{A / R}, R\right)=0$

Corollary. If $(R, m)$ is a complete local normal domain of dimension $n$ and $A$ is as above then $\mathrm{H}_{m}^{n}\left(\Omega_{A / R}\right)=0$.

This follows from local duality due to the fact that the canonical module of $R$ is isomorphic to a height 1 ideal in $R$ and $R$ can be embedded into this ideal.

2.2. Sub-Lemma. Let $R \hookrightarrow S$ be two integral domains such that $S=R\left[X_{1}, \ldots, X_{n}\right] /\left(f_{1}, \ldots, f_{n}\right)$, where $f_{1}, \ldots, f_{n}$ form an $R\left[X_{1}, \ldots, X_{n}\right]$ sequence and $Q(S) / Q(R)$ is separably algebraic. Then $\Gamma_{S / R}=0$.

Proof. Let $I=\left(f_{1}, \ldots, f_{n}\right)$. We have the following exact sequence of $S$-modules:

$0 \rightarrow \Gamma_{S / R} \rightarrow I / I^{2} \rightarrow \oplus_{1}^{n} S d x_{i} \rightarrow \Omega_{S / R} \rightarrow 0 \ldots \ldots \ldots \ldots \ldots \ldots$ (2)

Since $I / I^{2}$ is a free $S$ module of rank $n$ and $S$ is a domain, $I / I^{2}$ is torsion-free over $S$; hence so is $\Gamma_{S / R}$ unless it is 0 .

Since $Q(S) / Q(R)$ is separably algebraic, $\Omega_{Q(S) / Q(R)}=0$, i.e. $\Omega_{S / R} \otimes$ $Q(S)=0$. Tensoring the above sequence with $Q(S)$ we obtain the following exact sequence:

$0 \rightarrow Q(S) \otimes \Gamma_{S / R} \rightarrow Q(S) \otimes I / I^{2} \rightarrow \oplus_{1}^{n} Q(S) d x_{i} \rightarrow 0 \ldots \ldots \ldots$ (3)

Since rank $Q(S) \otimes I / I^{2}=n=\operatorname{rank} \oplus_{1}^{n} Q(S) d x_{i}$, it follows from (3) that $Q(S) \otimes \Gamma_{S / R}=0$. Hence $\Gamma_{S / R}=0$.

Lemma. Let $R \hookrightarrow S$ be two regular rings such that $S$ is a modulefinite extension of $R$ and $Q(S)$ is separable algebraic over $Q(R)$. Then $\Gamma_{S / R}=0$.

Proof. First we assume that both $R$ and $S$ are local.

Since $\operatorname{dim} R=\operatorname{dim} S$, we have $S \simeq R\left[X_{1}, \ldots, X_{n}\right] / P$ where $P$ is a prime ideal of height $n$ (one could also write $S=R\left[X_{1}, \ldots, X_{n}\right]_{T} / P$ where $T$ is the inverse image of the maximal ideal of $S$ under the onto map $\left.R\left[X_{1}, \ldots, X_{n}\right] \rightarrow S\right)$. Since $S$ is regular local, $P$ is a complete intersection ideal generated by $n$ elements and $P / P^{2}$ is a free $S$-module of rank $n$. The proof now follows by the above sub-lemma.

Now let us assume that $R$ is local. Let $m$ denote the maximal ideal of $R$ and let $m_{1}, \ldots, m_{r}$ denote the maximal ideals of $S$. Let $S_{i}=S_{m_{i}}$ for $1 \leq i \leq n$ and let $\widehat{R}, \widehat{S}$ and $\widehat{S}_{i}$ denote the $m$-adic completions of $R, S$ 
and $S_{i}$ respectively. Then $\widehat{S}_{i}$ is a complete regular local ring modulefinite over $\widehat{R}$. It can be checked that $\Gamma_{S / R} \otimes \widehat{S} \simeq \Gamma_{\widehat{S} / \widehat{R}} \simeq \bigoplus_{i=1}^{r} \Gamma_{\widehat{S_{i}} / \widehat{R}}$. Due to the result proved above it follows that $\Gamma_{S / R} \otimes \widehat{S}=0$ and hence $\Gamma_{S / R}=0$.

Finally the general case follows via localization.

2.3. Theorem ( André, Gabber-Ramero, Popescu, see introduction ) Let $A \rightarrow B$ be an extension of Noetherian rings such that $B$ is geometrically regular over $A$. Then $\Omega_{B / A}$ is $B$-flat and $\Gamma_{B / A}=0$.

Proof. Popescu's theorem ([11], [14]) asserts that in the above situation $B=\underline{\lim _{C}} C$, a filtered inductive limit over smooth $A$-algebras $C$. Then $\Omega_{C / A}$ is a finitely generated projective C-module and $\Gamma_{C / A}=0$. Since $\Omega_{B / A}=\underline{\lim _{C / A}} \Omega_{B / A}=\underline{\lim } \Gamma_{C / A}$, the conclusion follows.

2.4. Lemma. $A \rightarrow B \rightarrow C$ are injective ring homomorphisms of integral domains such that $Q(C)$ is a separable algebraic extension of $Q(B)$ and $\Omega_{B / A}$ is a flat $B$-module. Then the following sequence:

$$
0 \rightarrow \Omega_{B / A} \otimes_{B} C \rightarrow \Omega_{C / A} \rightarrow \Omega_{C / B} \rightarrow 0
$$

is exact.

Proof. we have an exact sequence:

$0 \rightarrow \Gamma_{C / B / A} \rightarrow \Omega_{B / A} \otimes_{B} C \rightarrow \Omega_{C / A} \rightarrow \Omega_{C / B} \rightarrow 0 \ldots \ldots \ldots . . .4$ (4)

Since $\Omega_{B / A}$ is $B$-flat, $\Omega_{B / A} \otimes_{B} C$ is $C$-flat; since $C$ is an integral domain this implies that $\Gamma_{C / B / A}$ is a torsion-free $C$-module unless it is null. Let $S=C-\{0\}$. Tensoring (4) with $S^{-1} C=Q(C)$ we obtain the following exact sequence:

$0 \rightarrow S^{-1} \Gamma_{C / B / A} \rightarrow \Omega_{Q(B) / Q(A)} \otimes Q(C) \rightarrow \Omega_{Q(C) / Q(A)} \rightarrow \Omega_{Q(C) / Q(B)} \rightarrow 0$

Since $Q(C)$ is separable algebraic over $Q(B), Q(C)$ is quasi-smooth (in Grothendieck's language: formally smooth with respect to discreet topology) over $Q(B)$ and hence it follows that $S^{-1} \Gamma_{C / B / A}=0$ (20.5.7, [5]). Since $\Gamma_{C / B / A}$ is torsion-free, it follows that $\Gamma_{C / B / A}=0$.

2.5. Here we state a result that has been used several times in this paper. This result is known as Jacobi-Zariski sequence, henceforth JZ sequence.

JZ sequence.([1], [14]) Given ring extensions $A \rightarrow B \rightarrow C$, there exists an exact sequence $\Gamma_{C / A} \rightarrow \Gamma_{C / B} \rightarrow \Omega_{B / A} \otimes C \rightarrow \Omega_{C / A} \rightarrow \Omega_{C / B} \rightarrow$ 0 . If $\Omega_{B / A}$ is $B$-flat then this sequence can be extended by putting $\Gamma_{B / A} \otimes C$ on the left.

For a proof we refer the reader to $([14])$. 

AND EXTENSION OF PURITY

3.1. Theorem. Let $(R, m)$ be a complete discrete valuation ring containing a field $k$ of characteristic 0 and let $B$ denote its integral closure in an algebraic extension $F$ of $Q(R)$. Then $\operatorname{Hom}_{R}\left(\Omega_{B / k}, R\right) \neq 0$.

Proof. Let $K$ denote the field of fractions of $R$. Since characteristic of $k=0, R$ is geometrically regular over $k$. By theorem $2.3, \Omega_{R / k}$ is a flat $R$-module and hence torsion-free over $R$. Let $R \subset C \subset C^{\prime} \subset B$ be such that $C, C^{\prime}$ are integral closures of $R$ in $Q(C), Q\left(C^{\prime}\right)$ respectively and $[Q(C): K]<\infty,\left[Q\left(C^{\prime}\right): K\right]<\infty$. Then $C, C^{\prime}$ are module-finite extensions of $R$ and $C^{\prime}$ is a module finite extension of $C$. Since $R$ is a complete d.v.r., $C, C^{\prime}$ are also the same; hence, by argument as above, $\Omega_{C / k}\left(\Omega_{C^{\prime} / k}\right)$ is a flat $C\left(C^{\prime}\right)$ module and $\Gamma_{C^{\prime} / C}=0$ (lemma 2.2). Due to lemma 2.4 and the JZ sequence 2.5 we obtain the following short exact sequences:

$$
\begin{aligned}
& 0 \rightarrow \Omega_{C / k} \otimes B \rightarrow \Omega_{B / k} \rightarrow \Omega_{B / C} \rightarrow 0 \ldots \ldots \ldots \ldots \ldots \ldots \ldots \ldots \ldots \\
& 0 \rightarrow \Omega_{R / k} \otimes C \rightarrow \Omega_{C / k} \rightarrow \Omega_{C / R} \rightarrow 0 \ldots \ldots \ldots \ldots \ldots \ldots \ldots \ldots \ldots \\
& 0 \rightarrow \Omega_{C / k} \otimes C^{\prime} \rightarrow \Omega_{C^{\prime} / k} \rightarrow \Omega_{C^{\prime} / C} \rightarrow 0 \text {. } \\
& 0 \rightarrow \Omega_{C / R} \otimes C^{\prime} \rightarrow \Omega_{C^{\prime} / R} \rightarrow \Omega_{C^{\prime} / C} \rightarrow 0
\end{aligned}
$$

Also note that $\Omega_{B / k}=\underset{\longrightarrow}{\lim } \Omega_{C / k}$ and $\Omega_{B / R}=\underset{\longrightarrow}{\lim } \Omega_{C / R}$ over all $\mathrm{C}$ as above

Since characteristic of $k=0$ and $R$ is a complete d.v.r., any $C$ as above is of the form $C=R[X] /(f(X))=R[b]$ where $f(X)$ is monic irreducible in $R[X]([13])$. Thus $C$ is a free $R$-module with bases $1, b, b^{2}, \ldots, b^{n-1}, n=\operatorname{deg} f(X)$. Hence $\Omega_{C / R} \simeq C /\left(f^{\prime}(b)\right) d b$ is a cyclic $C$-module generated by $d b$. It follows from (5), (6), (7) and (9) that any $\omega \in \Omega_{B / k}$ can be expressed as:

$\omega=\Sigma t_{i} \omega_{i}+c d b, c \in C$ for some $C$ as above, $\omega_{i} \in \Omega_{R / k} t_{i} \in C$,

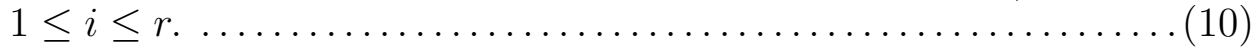

Let $\operatorname{Tr}: F \rightarrow K$ denote the trace-map: for any $x \neq 0 \in F$, if $x \in \mathrm{L} \subset F$ such that $[\mathrm{L}: K]<\infty$, then $\operatorname{Tr}(x)=\operatorname{Tr}_{\mathrm{L} / K}(x) /[\mathrm{L}: K]$. Let $\mathrm{D} \in \operatorname{Der}_{k}(R)$, be non-null (e.g. $D=\partial / \partial X, R=\mathbb{k}[[X]], \mathbb{k}=$ $R / m)$. Since characteristic of $k=0, \mathrm{D}$ can be extended uniquely to a derivation $\mathrm{D}$ (same notation): $B \rightarrow F$. We define an $R$-linear map $\mathrm{L}: \Omega_{B / k} \rightarrow K$ as follows: for any $\omega \in \Omega_{B / k}, \mathrm{~L}(\omega)=\operatorname{Tr}(\tilde{\mathrm{D}}(\omega))$, where $\tilde{\mathrm{D}}: \Omega_{B / k} \rightarrow F$ is the $B$-linear map induced by $\mathrm{D}$.

Claim. Im $\mathrm{L} \subset R$ 
Proof of the Claim. Due to (10), given any $\omega \in \Omega_{B / k}$, we have $\mathrm{L}(\omega)=\mathrm{L}\left(\Sigma t_{i} \omega_{i}\right)+\mathrm{L}(c d b)$, For any $x \in B, \operatorname{Tr}(x) \in R$ and $\tilde{\mathrm{D}}\left(\omega_{i}\right) \in R$ for every $i$.

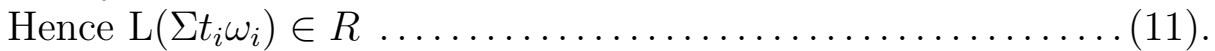

We need to show that $\mathrm{L}(c d b) \in R$. Recall that $f(b)=0$ where $f(X)$ is an irreducible polynomial in $R[X]$. Let $f(X)=X^{n}+r_{1} X^{n-1}+\cdots+$ $r_{n}, r_{i} \in R$. Then we have $f^{\prime}(b) d b+\Sigma_{1}^{n} d\left(r_{i}\right) b^{n-i}=0$.

Hence $f^{\prime}(b) \tilde{\mathrm{D}}(d b)+\Sigma \mathrm{D}\left(r_{i}\right) b^{n-i}=0$.

Due to separability $f^{\prime}(b) \neq 0$. This implies

$$
\tilde{\mathrm{D}}(d b)=-\Sigma \mathrm{D}\left(r_{i}\right) b^{n-i} / f^{\prime}(b), 1 \leq i \leq n .
$$

Since $c \in R[b], c=\Sigma s_{i} b^{i}, s_{i} \in R, 0 \leq i \leq n-1$. Due to the fact that $f(b)=0$ and $\mathrm{D}\left(r_{i}\right) \in R$ for $1 \leq i \leq n$, we obtain

$$
\tilde{\mathrm{D}}(c d b)=c \tilde{\mathrm{D}}(d b)=\Sigma \mu_{i} b^{i} / f^{\prime}(b), \mu_{i} \in R, 0 \leq i \leq n-1 .
$$

Since $\operatorname{Tr}\left(b^{i} / f^{\prime}(b)\right)=0$ for $i<n-1$ and $\operatorname{Tr}\left(b^{n-1} / f^{\prime}(b)\right)=1$ ([13]), we have $\mathrm{L}(c d b)=\Sigma \mu_{i} \operatorname{Tr}\left(b^{i} / f^{\prime}(b)\right) \in R \ldots \ldots \ldots \ldots \ldots \ldots \ldots \ldots \ldots \ldots \ldots \ldots \ldots \ldots$ (12)

From (11) and (12) $\mathrm{L}(\omega) \in R$ for any $\omega \in \Omega_{B / k}$ and thus the claim is proved.

3.2 Remarks. Notations are as in the proof of the above theorem.

1. We have $\Omega_{B / k}=\lim _{C / k}$ and $\Gamma_{B / k}=\lim _{C / k}$. Since $C$ is complete d.v.r. containing a field $k$ of characteristic $0, C$ is geometrically regular over $k$. It follows by theorem 2.3 that $\Omega_{C / k}$ is a flat torsion free $C$-module and $\Gamma_{C / k}=0$. Hence $\Omega_{B / k}$ is a flat torsion free $B$-module and $\Gamma_{B / k}=0$.

2. We have $\Omega_{B / R}=\lim _{C / R}$. Since $\Omega_{C / R}$ is a cyclic $C$-module of finite length, $\mathrm{H}_{m}^{0}\left(\Omega_{B / R}\right)=\Omega_{B / R}$ and by lemma $2.1 \mathrm{H}_{m}^{1}\left(\Omega_{B / R}\right)=0$.

3.3 Corollary. Notations are as in the proof of the above theorem.

The following sequence $0 \rightarrow \Omega_{B / R} \rightarrow \Omega_{R / k} \otimes \mathrm{H}_{m}^{1}(B) \rightarrow \mathrm{H}_{m}^{1}\left(\Omega_{B / k}\right) \rightarrow$ 0 is exact.

From Remark 1 and the exact sequence: $0 \rightarrow \Omega_{R / k} \otimes B \rightarrow \Omega_{B / k} \rightarrow$ $\Omega_{B / R} \rightarrow 0$ we obtain the following exact sequence:

$0 \rightarrow \mathrm{H}_{m}^{0}\left(\Omega_{B / R}\right) \rightarrow \mathrm{H}_{m}^{1}\left(\Omega_{R / k} \otimes B\right) \rightarrow \mathrm{H}_{m}^{1}\left(\Omega_{B / k}\right) \rightarrow 0$.

Since $\Omega_{R / k}$ is a flat $R$-module, we have $\mathrm{H}_{m}^{1}\left(\Omega_{R / k} \otimes B\right) \simeq \Omega_{R / k} \otimes$ $\mathrm{H}_{m}^{1}(B)$.

The assertion now follows from Remark 2 .

3.4. Next we deal with the non-complete d.v.r. case.

Proposition. Let $(R, m)$ be a discreet valuation ring containing a field $k$ of characteristic 0 with a non-null derivation $\mathrm{D} \in \operatorname{Der}_{k}(R)$ and 
let $B$ be its integral closure in an algebraic extension $F$ of $Q(R)$. Then $\operatorname{Hom}_{R}\left(\Omega_{B / k}, R\right) \neq 0$.

Proof. Let $K$ denote the field of fractions of $R$. Since characteristic of $k=0$, D can be extended uniquly to a derivation $\mathrm{D}$ (same notation): $B \rightarrow F$. Let $\operatorname{Tr}: F \rightarrow K$ denote the trace map. We define an $R$-linear map $\mathrm{L}: \Omega_{B / k} \rightarrow K$ in the following way:

for any $\omega \in \Omega_{B / k}, \mathrm{~L}(\omega)=\operatorname{Tr}(\tilde{\mathrm{D}}(\omega))$, where $\tilde{\mathrm{D}}: \Omega_{B / k} \rightarrow F$ is the $B$-linear map induced by $D$.

Claim. Im L $\subset R$.

Can assume $\tilde{\mathrm{D}}(\omega) \neq 0$. We will show that $\mathrm{L}(\omega) \in R$. Let $C$ be a normal domain integral over $R$ such that $[Q(C): K]<\infty$ and $C \subset B$. We have $\Omega_{B / k}=\lim _{C / k}$ over all such $C$ as above. For any $R \subset C \subset C^{\prime}, C, C^{\prime}$ as above are module finite extensions of $R$ and hence are semi-local Dedekind domains. Since characteristics of $k=0$, for any such $C, k \hookrightarrow C$ is geometrically regular and hence $\Omega_{C / k}$ is a flat $C$-module (theorem 2.3). The exact sequences (5)-(8) in 3.1 are valid here. In particular we have the following exact sequence:

$$
0 \rightarrow \Omega_{C / k} \otimes C^{\prime} \rightarrow \Omega_{C^{\prime} / k} \rightarrow \Omega_{C^{\prime} / C} \rightarrow 0
$$

The left exactness in (13) follows from lemma 2.2 and the JZ sequence 2.5. Without any lose of generality can assume $\omega \in \Omega_{C / k}$ for some $C$ as above. Let $Q_{1}, \ldots, Q_{r}$ be the maximal ideals of $C$ lying over $m$. Then $\widehat{C} \simeq \widehat{C}_{Q_{1}} \times \cdots \times \widehat{C}_{Q_{r}}$ (m-adic completion) $\ldots \ldots \ldots \ldots \ldots \ldots \ldots$ (14)

Here each $\widehat{C}_{Q_{i}}$ is a complete d.v.r. and a module finite extension of $\widehat{R}$. Since $R$ contains a field of characteristic 0 , each $C_{Q_{i}} \rightarrow \widehat{C}_{Q_{i}}$ is geometrically regular and hence so is $C \rightarrow \widehat{C}$. By theorem $2.3 \Omega_{\widehat{C} / C}$ is a flat $\widehat{C}$-module and $\Gamma_{\widehat{C} / C}=0$. Hence, due to the JZ sequence 2.5, we obtain the following short exact sequence:

$0 \rightarrow \Omega_{C / k} \otimes \widehat{C} \rightarrow \Omega_{\widehat{C} / k} \rightarrow \Omega_{\widehat{C} / C} \rightarrow 0$

We also have $\Omega_{\widehat{C} / k} \simeq \Omega_{\widehat{C}_{Q_{1} / k}} \times \cdots \times \Omega_{\widehat{C}_{Q_{r}} / k}$

Due to the fact that $\Omega_{B / k}=\underline{\lim } \Omega_{C / k}$ and (13), (15) and (16) we can write

$$
\omega=\omega_{1} \times \cdots \times \omega_{r}, \omega_{i} \in \Omega_{\widehat{C}_{Q_{i} / k}}, 1 \leq i \leq r .
$$

Since $\mathrm{D} \in \operatorname{Der}_{k}(R)$ and $\operatorname{Der}_{k}(R, \widehat{R}) \simeq \operatorname{Der}_{k}(\widehat{R})$ ( $R$ being local any such $\mathrm{D}$ is continuous), D can be extended uniquely to a derivation $\widehat{\mathrm{D}}: \widehat{R} \rightarrow \widehat{R}$. This derivation $\widehat{\mathrm{D}}$ can be extended uniquely to a derivation $\widehat{\mathrm{D}}_{i}: \widehat{C}_{Q_{i}} \rightarrow K_{i}$, where $K_{i}$ is the field of fractions of $\widehat{C}_{Q_{i}}, 1 \leq i \leq r$. Thus $\mathrm{D}$ can be extended uniquely to a derivation $\mathrm{D}^{\prime}: \widehat{C} \rightarrow K_{1} \times \cdots \times K_{r}$ 
such that $\mathrm{D}^{\prime} \mid \widehat{C}_{Q_{i}}=\widehat{\mathrm{D}}_{i}$. Due to uniqueness of extension of derivation we have $\mathrm{D}^{\prime} \mid C=D$.

Hence from $(17)$ we have $\tilde{\mathrm{D}}(\omega)=\Sigma \widetilde{\widehat{\mathrm{D}}}_{i}\left(\omega_{i}\right)$ where $\tilde{\widehat{\mathrm{D}}}_{i}$ is the corresponding $\widehat{C}_{Q_{i}}$-linear map: $\Omega_{\widehat{C}_{Q_{i}} / k} \rightarrow K_{i}$. This implies that $L(\omega)=$ $\operatorname{Tr}(\tilde{\mathrm{D}}(\omega))=\Sigma \operatorname{Tr}\left(\widetilde{\widehat{\mathrm{D}}}_{i}\left(\omega_{i}\right)\right)$. By theorem 3.1, $\operatorname{Tr}\left(\tilde{\widehat{\mathrm{D}}}_{i}\left(\omega_{i}\right)\right) \in \widehat{R}, 1 \leq i \leq r$. Hence $L(\omega) \in K \cap \widehat{R}=R$ and the claim is proved.

Remark. Remarks in 3.2 and corollary of 3.3 are also valid here.

3.5. Our next theorem in this section addresses the general set-up.

Theorem. Let $R$ be a normal domain containing a field $k$ of characteristic 0 with a non-null derivation $\mathrm{D} \in \operatorname{Der}_{k}(R)$ and let $B$ be its integral closure in an algebraic extension $F$ of $Q(R)$. Then $\operatorname{Hom}_{R}\left(\Omega_{B / k}, R\right)$ $\neq 0$. In particular if $(R, m)$ is a complete local normal domain of dimension $n$ containing a field $k$ of characteristic 0 with a derivation as above, then $\mathrm{H}_{m}^{n}\left(\Omega_{B / k}\right) \neq 0$.

Proof. Let $K$ denote the field of fractions of $R$. Since characteristic of $k=0, \mathrm{D}$ can be extended to a derivation $\mathrm{D}$ (same notation): $B \rightarrow$ $F$ and hence to a derivation: $B_{P} \rightarrow F$ for any prime ideal $P$ of $R$. Let $\operatorname{Tr}: F \rightarrow K$ denote the trace map. We define an $R$-linear map $\mathrm{L}: \Omega_{B / k} \rightarrow K$ in the following way:

for any $\omega \in \Omega_{B / k}, \mathrm{~L}(\omega)=\operatorname{Tr}(\tilde{\mathrm{D}}(\omega))$ where $\tilde{\mathrm{D}}: \Omega_{B / k} \rightarrow F$ is the $B$ linear map induced by $\mathrm{D}$; the same prescription works for $\Omega_{B_{P} / k} \rightarrow F$ for any prime ideal $P$ of $R$.

Claim. Im $\mathrm{L} \subset R$.

Can assume $\tilde{\mathrm{D}}(\omega) \neq 0$. Let $P$ be a prime ideal of height 1 in $R$. Let $\omega$ also denote the image of $\omega$ in $\Omega_{B_{P} / k}$. It follows from proposition 3.4 that $\mathrm{L}(\omega) \in R_{P}$.

Since this holds for every prime ideal $P$ of height 1 in $R$ and $R$ is a normal domain, $\mathrm{L}(\omega) \in \underset{h t P=1}{\cap} R_{P}=R$. Hence the claim follows.

In particular if $R$ is a complete local normal domain then the canonical module of $R$ is isomorphic to a height 1 ideal of $R$. Hence the assertion follows by local duality.

3.6. The following theorem deals with complete local domains. We recall that if $(C, q)$ is a complete local domain of dimension $n$ with coefficient ring $V(k)$, then $\Omega_{C / V}\left(\Omega_{C / k}\right)$ is not necessarily a finitely generated $C$-module.

Theorem. Let $(C, q)$ be a complete local domain of dimension $n \geq 2$ with coefficient ring $V$ (field $k$ ). Let $(R, m)$ be a power series ring over $V(k)$ contained in $C$ such that $C$ is a module finite extension of $R$. We 
have the following:

i) if $Q(C) / Q(R)$ is separably algebraic, then rank $\widehat{\Omega}_{C / k}=n$, rank $\widehat{\Omega}_{C / V}=n-1$ and $\mathrm{H}_{q}^{n}\left(\Omega_{C / V}\right)\left(\mathrm{H}_{q}^{n}\left(\Omega_{C / k}\right)\right) \neq 0$,

ii) if characteristic of $k=p>0, k$ perfect and $\Omega_{Q(C) / k} \neq 0$, then $\operatorname{rank} \widehat{\Omega}_{C / k}=\left[Q(C): Q(C)^{p}\right], \mathrm{H}_{q}^{n}\left(\Omega_{C / k}\right) \neq 0$ and

iii) if $Q(C) / Q(R)$ is separably algebraic, then $\mathrm{H}_{q}^{n}\left(\Omega_{C / R}\right)=0$; moreover if $\Omega_{C / R} \neq 0$ and $C$ is normal, then $\mathrm{H}_{q}^{n-1}\left(\Omega_{C / R}\right) \neq 0$.

Proof. Let $R=k\left[\left[X_{1}, \ldots, X_{n}\right]\right]$ or $R=V\left[\left[X_{1}, \ldots, X_{n-1}\right]\right]$ be contained in $C$ such that $C$ is a module finite extension of $R$. Let $m$ denote the maximal ideal of $R$. Then for any $C$-module $M, \mathrm{H}_{m}^{i}(M)=\mathrm{H}_{q}^{i}(M)$ for $i \geq 0$. We intend to show that $\widehat{\Omega}_{C / V}\left(\widehat{\Omega}_{C / k}\right)$, the $m$-adic, equivalently $q$-adic, completion of $\Omega_{C / V}\left(\Omega_{C / k}\right)$, is a finitely generated $C$-module of finite rank and hence a finitely generated $R$-module of finite rank. This would imply that $\operatorname{Hom}_{R}\left(\widehat{\Omega}_{C / V}\left(\widehat{\Omega}_{C / k}\right), R\right) \neq 0$. Since $R$ is complete we have

$$
\operatorname{Hom}_{R}\left(\Omega_{C / V}\left(\Omega_{C / k}\right), R\right)=\operatorname{Hom}_{R}\left(\widehat{\Omega}_{C / V}\left(\widehat{\Omega}_{C / k}\right), R\right) \neq 0
$$

Now the result would follow by local duality.

i) a) Equicharacteristic case. The arguments in $(21.9 .5,[5])$ show that $\widehat{\Omega}_{C / k}$ is a finitely generated $C$-module and $\operatorname{rank}_{C} \widehat{\Omega}_{C / K}=n$.

b) Mixed characteristic p. In this case $C$ is a module finite extension of $R=V\left[\left[X_{1}, \ldots, X_{n-1}\right]\right]$. We need to modify the arguments in (21.9.5, $[5])$. For this purpose we need the following lemma.

Lemma. Let $(R, m)$ be as above. Then $\Omega_{R / V}$ is a faithfully flat $R$-module, $\widehat{\Omega}_{R / V} \simeq R^{n-1}$ and the sequence

$$
0 \rightarrow \cap_{t \geq 0}^{t} \Omega_{R / V} \rightarrow \Omega_{R / V} \rightarrow \widehat{\Omega}_{R / V} \rightarrow 0
$$

is split exact.

Proof of the Lemma. There exists an exact sequence:

$$
\begin{aligned}
0 \rightarrow R / m & \rightarrow m / m^{2} \rightarrow \Omega_{R / V} \otimes R / m \rightarrow 0 \\
\overline{1} & \rightarrow \bar{p}
\end{aligned}
$$

This implies that $\operatorname{dim}\left(\Omega_{R / V} \otimes R / m\right)=n-1$. Hence, due to theorem 2.3, $\Omega_{R / V}$ is a faithfully flat $R$-module. Moreover $T$, the Hausdorffmodule associated with $\Omega_{R / V}$, i.e. $\Omega_{R / V} / \cap m^{t} \Omega_{R / V}$, is generated by images of $d X_{1}, \ldots, d X_{n-1}$ over $R$. $R$ being complete implies $T$ is also complete and hence $T=\widehat{\Omega}_{R / V}$. Due to the existence of derivations $\partial / \partial X_{i}: R \rightarrow R, \partial / \partial X_{i}\left(X_{j}\right)=\delta_{i j}, 1 \leq i, j \leq n-1$, and duality between 
derivatives and differentials it follows that images of $d X_{1}, \ldots, d X_{n-1}$ are linearly independent in $\widehat{\Omega}_{R / V}$. Thus $\widehat{\Omega}_{R / V}$ is free $R$-module of rank $n-1$. And this implies the split exactness of the sequence in the lemma.

Now arguing as in $(21.9 .5,[5])$ we observe that $\widehat{\Omega}_{C / V}$ is a finitely generated $C$-module of finite rank $n-1$ and hence a finitely generated $R$-module of finite rank.

ii) Characteristic of $k=p>0$. We assume that $k$ is perfect and $\Omega_{Q(C) / k} \neq 0$. Let $R_{1}=k\left[\left[X_{1}^{p}, \ldots, X_{n}^{p}\right]\right]$. By $(21.9 .4,[5]) \widehat{\Omega}_{C / k}=\Omega_{C / R_{1}}$. let $A=R_{1}\left[C^{p}\right]$. Then $\Omega_{C / R_{1}}=\Omega_{C / A}$. Let $y_{1}, \ldots, y_{t}$ in $C$ form a $p$-basis of $Q(C)$ over $Q\left(R_{1}\right)$ i.e. $Q(C)$ over $k$, since $k$ is perfect. Due to our assumption that $\Omega_{Q(C) / k} \neq 0$ such a basis exists. Let $B=A\left[y_{1}, \ldots, y_{t}\right]$. Then $B$ is a free A-module of rank $p^{t}$ and $\Omega_{B / A}$ is a free B-module of rank t with basis $d y_{1}, \ldots, d y_{t}$. We have the following short exact sequence:

$$
\Omega_{B / A} \otimes C \rightarrow \Omega_{C / A} \rightarrow \Omega_{C / B} \rightarrow 0
$$

Since $Q(C)=Q(B), \Omega_{C / B}$ is a torsion C-module.

Claim. $d y_{1}, \ldots, d y_{t}$ are also linearly independent in $\Omega_{C / A}$.

The Claim follows via the existence of linearly independent $Q(A)$ derivations $\partial / \partial y_{i}, Q(C) \rightarrow Q(C), \partial / \partial y_{i}\left(y_{j}\right)=\delta_{i j}, 1 \leq i, j \leq t$.

Due to the above exact sequence we conclude that $\Omega_{C / A}$ is a finitely generated $C$-module of rank $t$. Hence $\widehat{\Omega}_{C / k}$ is a finitely generated $R$ module of finite rank.

The referee made the following observation: if $Q(C) / Q(R)$ is not separable, then since $R \rightarrow C$ s module-finite,

$$
\Omega_{C / R} \otimes Q(C) \simeq \Omega_{C / R} \otimes Q(C) \simeq \Omega_{Q(C) / Q(R)} \neq 0 .
$$

Since $\widehat{\Omega}_{C / k} \rightarrow \widehat{\Omega}_{C / R}$ is onto, it follows that $\widehat{\Omega}_{C / k} \otimes Q(C) \neq 0$ and thus $\widehat{\Omega}_{C / k}$ has a positive rank.

iii) Now assume that $R$ is a complete regular local ring contained in $C$ such that $C$ is a module finite extension of $R$ and $Q(C) / Q(R)$ is separably algebraic. Then by corollary to lemma $2.1, H_{m}^{n}\left(\Omega_{C / R}\right)=0$.

Assume in addition that $\Omega_{C / R} \neq 0$ and $C$ is normal.

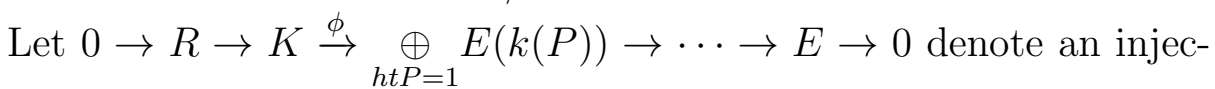
tive resolution of $R, K=Q(R)$. Let $T=\operatorname{Im} \phi$; then $T \hookrightarrow \underset{h t P=1}{\oplus} E(k(P))$ is an essential extension.

Consider the exact sequence: $0 \rightarrow R \rightarrow K \rightarrow T \rightarrow 0$. By assumption $\operatorname{Hom}_{R}\left(\Omega_{C / R}, K\right)=\operatorname{Hom}_{K}\left(\Omega_{(Q(C) / K}, K\right)=0$ and $\operatorname{Ext}_{R}^{i}\left(\Omega_{C / R}, K\right)=0$ for every $i>0$. Hence $\operatorname{Hom}_{R}\left(\Omega_{C / R}, T\right) \simeq \operatorname{Ext}_{R}^{1}\left(\Omega_{C / R}, R\right)$. Thus, by 
local duality, in order to prove our assertion we need to show that $\operatorname{Hom}_{R}\left(\Omega_{C / R}, T\right) \neq 0$.

We have an injection:

$$
\operatorname{Hom}_{R}\left(\Omega_{C / R}, T\right) \hookrightarrow \operatorname{Hom}_{R}\left(\Omega_{C / R}, \underset{h t P=1}{\oplus} E(k(P))\right) .
$$

If $\operatorname{Hom}_{R}\left(\Omega_{C / R}, E(k(P))\right)=\operatorname{Hom}_{R_{P}}\left(\Omega_{C_{P} / R_{P}}, E(k(P))\right)=0$ for every prime ideal $P$ of $R$ of height 1 , then $\Omega_{C_{P} / R_{P}}=0$ for every prime ideal of height 1 . Hence $\Omega_{C_{q} / R}=0$ for every prime ideal $q$ of height 1 in $C$. By the theorem on purity of branch locus ([8], [13]) this would imply $\Omega_{C / R}=0$, a contradiction. Thus there exists at least one prime ideal $P$ of height 1 such that $\operatorname{Hom}_{R}\left(\Omega_{C / R}, E(k(P))\right) \neq 0$. Hence $\operatorname{Hom}_{R}\left(\Omega_{C / R}, \underset{h t P=1}{\oplus} E(k(P))\right) \neq 0$.

Let $f(\neq 0) \in \operatorname{Hom}_{R}\left(\Omega_{C / R}, \underset{h t P=1}{\oplus} E(k(P))\right)$. $\Omega_{C / R}$ is a finitely generated $R$-module. Let $\omega_{1}, \ldots, \omega_{t}$ denote the generators of $\Omega_{C / R}$; then each $f\left(\omega_{i}\right), 1 \leq i \leq t$ has only finitely many non-null components in $\underset{h t P=1}{\oplus} E(k(P))$. Since $T \hookrightarrow \underset{h t P=1}{\bigoplus} E(k(P))$ is essential, there exists $s \neq 0$ in $R$ such that $s f\left(\omega_{i}\right) \in T$ for $1 \leq i \leq t$ and $s f\left(\omega_{i}\right) \neq 0$ for at least one $i$. Thus $\operatorname{Hom}_{R}\left(\Omega_{C / R}, T\right) \neq 0$.

Remarks. 1) The proof for part i) of the above theorem is valid in equicharacteristic when dimension $C=1$.

2) With hypothesis as in part iii) of the above theorem, $\operatorname{dim} \Omega_{C / R}=$ $n-1$. The proof in this part is also valid in the non-complete case.

3.7. Our final result of this section can be viewed as an extension of the theorem on purity of branch locus ([10], [15]) to infinitely generated integrally closed domains $B$ integral over a regular local ring $R$. Let us recall that an extension $R \rightarrow S$ is unramified if and only if $\Omega_{S / R}=0$ and a local extension $(R, m) \rightarrow(S, q)$ of essentially finite type is unramified if and only if $m S=q$ and $S / q$ is a separable algebraic extension of $R / m$.

Theorem. Let $R$ be a regular local ring and let $B$ denote the integral closure of $R$ in a field extension $K$ of $Q(R)$ such that $K / Q(R)$ is separably algebraic. Suppose that for every prime ideal $q$ of height 1 in $B, \Omega_{B_{q} / R}=0$. Then $\Omega_{B / R}=0$ i.e. $B$ is an unramified extension of $R$.

Proof. If $[K: Q(R)]<\infty$ then this is the usual theorem on purity of branch locus. Assume that $[K: Q(R)]=\infty$. Let $R \subset C \subset C^{\prime} \subset B$ be such that $C, C^{\prime}$ are integral closures of $R$ in $Q(C), Q\left(C^{\prime}\right)$ respectively and $[Q(C): Q(R)]<\infty,\left[Q\left(C^{\prime}\right): Q(R)\right]<\infty$. Then $C, C^{\prime}$ are modulefinite extensions of $R$ and $C^{\prime}$ is a module finite extension of $C$. Since 
$B=\underline{\lim C}, \Omega_{B / R}=\underline{\lim }_{\longrightarrow} \Omega_{C / R}$ over all such $C$ as mentioned above. Let $q$ be a prime ideal of $B$ of height 1 and let $P=q \cap R$. Then $\Omega_{B_{P} / R}=\underset{\lim }{\longrightarrow} \Omega_{C_{P} / R}$. From the assumption it follows that $\Omega_{B_{P} / R}=0$. Each $C_{P}$ is a semi-local Dedekind domain and $C_{P} \hookrightarrow C_{P}^{\prime}$ is a module finite extension. Hence $\Gamma_{C_{P}^{\prime}} / C_{P}=0$ (lemma 2.2). By the JZ sequence 2.5 we obtain an exact sequence

$0 \rightarrow \Omega_{C_{P} / R} \otimes C_{P}^{\prime} \rightarrow \Omega_{C_{P}^{\prime} / R} \rightarrow \Omega_{C_{P}^{\prime} / C_{P}} \rightarrow 0$

Since $\Omega_{B_{P} / R}=0$, from the above exact sequence it follows that $\Omega_{C_{P} / R}=0$ for every such $C$. By our assumption this holds for every prime ideal $P$ of height 1 in $R$ and hence for prime ideals $\tau$ of height 1 in $C$ we have $\Omega_{C_{\tau / R}}=0$. Due to the theorem on purity of branch locus it follows that $\Omega_{C / R}=0$ for every such $C$. Hence $\Omega_{B / R}=0$.

\section{SECTION 4: Mixed CHARACTERISTIC}

In this section we deal with the situation $R \hookrightarrow B$ where $B$ is the absolute integral closure of $R$. In the positive characteristic $p>0$ case we have $\Omega_{B / R}=0, \Omega_{B / k}=0$, i.e. $k \rightarrow B, R \rightarrow B$ are unramified extensions. Due to theorem 3.5 we now concentrate on the mixed characteristic case. First we define formally unramified extension (19.10, $[5])$.

Definition. Let $f: A \rightarrow B$ be a continuous ring homomorphism between two topological rings. We say $B$ is formally unramified over $A$ if given any discrete $A$-algebra $C$, any ideal $I$ of $C$ such that $I^{2}=0, C / I$ with discrete topology and $\eta: C \rightarrow C / I$, the natural surjection, every continuous $A$-algebra homomorphism $\phi: B \rightarrow C / I$ has at most one $A$-algebra extension $\psi: B \rightarrow C$ such that $\phi=\eta$. $\psi$.

4.1. Theorem. Let $(R, m)$ be a complete regular local ring of dimension $\mathrm{n}$ in mixed characteristic $p$ with coefficient ring $V$ and let $B$ denote its absolute integral closure. We have the following:

i) $\Omega_{B / V}=p \Omega_{B / V}, \Omega_{B / R}=p \Omega_{B / R}$. These observations imply the following:

a) For any ideal $I$ of $R$ of the form $I=\left(p, x_{1}, . ., x_{i-1}\right)$ generated by a part of a system of parameters containing $p$ of $R, H_{I}^{i}\left(\Omega_{B / V}\right)=0$ and $H_{I}^{i}\left(\Omega_{B / R}\right)=0$. In particular $H_{m}^{n}\left(\Omega_{B / V}\right)=0$ and $H_{m}^{n}\left(\Omega_{B / R}\right)=0$.

b) $\widehat{\Omega}_{B / V}=0=\widehat{\Omega}_{B / R}$, where $(\widehat{-})$ denotes the I-adic completion. And hence $V \rightarrow B, R \rightarrow B, V \rightarrow \widehat{B}$ and $R \rightarrow \widehat{B}$ are all formally unramified extensions.

ii) Non-vanishing of $\mathrm{H}_{m}^{n-1}\left(\Omega_{B / R}\right)$ implies the direct summand property for integral extensions of $R([5])$ (equivalently descent of flatness 
for integral extensions of Noetherian rings $([10]))$ and the converse is also true.

Non-vanishing of $\mathrm{H}_{m}^{n-1}\left(\Omega_{B / V}\right)$ implies the direct summand property for integral extensions of $R$.

Proof. i) Actually we would prove the following more general statement:

Let $A$ be an integral domain of characteristic 0 and let $B$ be the absolute integral closure of an integral domain $C$ containing $A$. Let $t$ be a positive integer such that $t$ is not a unit in $C$. Then for any proper ideal $I$ of $A$ containing $t$ and for every $n>0$ we have $\Omega_{B / A}=$ $I^{n} \Omega_{B / A}$. Let $\widehat{\Omega}_{B / A}$ and $\widehat{B}$ denote the $I$-adic completion of $\Omega_{B / A}$ and $B$ respectively. Then $\widehat{\Omega}_{B / A}=0=\widehat{\Omega}_{\widehat{B} / A}$ and $A \rightarrow B, A \rightarrow \widehat{B}$ are formally unramified extensions with respect to the $I$-adic topology.

Proof. For every element $x(\neq 0)$ in $B, x$ has a $t^{n} t h$ root $y$ in $B$ i.e. $y^{t^{n}}=x$. Hence $d x=t^{n} y^{t^{n}-1} d y$; this implies that $\Omega_{B / A}=t^{n} \Omega_{B / A}$ and thereby $\Omega_{B / A}=I^{n} \Omega_{B / A}$ for every $n>0$.

Let $\tilde{B}=B / I^{n} B, \tilde{A}=A / I^{n} A$; via base change we obtain

$$
\Omega_{B / A} \otimes \tilde{B} \simeq \Omega_{\tilde{B} / \tilde{A}}
$$

We have from above, $\Omega_{\tilde{B} / \tilde{A}}=0$. Hence $\widehat{\Omega}_{B / A}=0$. Let $\widetilde{\widehat{B}}=\widehat{B} / I^{n} \widehat{B}=$ $B / I^{n} B$; this implies $\Omega_{\tilde{\hat{B}} / \tilde{A}}=\Omega_{\tilde{B} / \tilde{A}}=0$. Hence $\widehat{\Omega}_{\widehat{B} / A}=0$. It follows from (20.7.4 and 20.7.5, [5]) that $A \rightarrow B, A \rightarrow \widehat{B}$ are formally unramified extensions.

Next let $(A, q)$ be a complete local normal domain of dimension $n$ with coefficient ring $V$ and let $B$ be its absolute integral closure. By our assumption it follows from above that $\Omega_{B / V}=p^{t} \Omega_{B / V}$ and if $I=$ $\left(p, x_{1}, \ldots, x_{i-1}\right)$ generated by a part of a system of parameters of $R$ and $I_{t}=\left(p^{t}, x_{1}^{t}, . ., x_{i-1}^{t}\right)$, then $\Omega_{B / V}=I_{t} \Omega_{B / V}$ for $t>0$. Similar statement is valid for $\Omega_{B / R}$. Now the assertion follows immediately.

ii) Let $\alpha: R \hookrightarrow B$ denote the inclusion as mentioned in the setup. We need to show that $\alpha$ splits as an $R$-module map $\Leftrightarrow \mathrm{H}_{m}^{n-1}\left(\Omega_{B / R}\right) \neq 0$.

Recall that $\alpha$ splits as an $R$-module map $\Leftrightarrow B^{*}=\operatorname{Hom}_{R}(B, R) \neq 0$ i.e. $\mathrm{H}_{m}^{n}(B) \neq 0([8])$ (same condition for descent of flatness $([12])$ ).

First we deal with the unramified case i.e. $R=V\left[\left[X_{1}, \ldots, X_{n-1}\right]\right]$. If dimension of $R=1, R=V, \Omega_{Q(B) / Q(R)}=0$ implies that $\Omega_{B / R}[1 / p]=0$; hence $\mathrm{H}_{m}^{0}\left(\Omega_{B / R}\right)=\Omega_{B / R} \neq 0$.

We can assume $\operatorname{dim} R \geq 2$. 
Since the direct summand property is valid over $R([2])$, we have $\mathrm{H}_{m}^{n}(B) \neq 0$. Since $V \rightarrow R$ is geometrically regular, $\Omega_{R / V}$ is a flat $R$-module (theorem 2.3).

By lemma 2.4 we have the following short exact sequence:

$$
0 \rightarrow \Omega_{R / V} \otimes_{R} B \rightarrow \Omega_{B / V} \rightarrow \Omega_{B / R} \rightarrow 0
$$

Since $\Omega_{R / V}$ is a flat $R$-module, $\mathrm{H}_{m}^{i}\left(\Omega_{R / V} \otimes B\right) \simeq \Omega_{R / V} \otimes \mathrm{H}_{m}^{i}(B)$ for every $i>0$.

Applying local cohomology to the above short exact sequence, by i), we obtain the following short exact sequence:

$$
\rightarrow \mathrm{H}_{m}^{n-1}\left(\Omega_{B / R}\right) \rightarrow \Omega_{R / V} \otimes_{R} \mathrm{H}_{m}^{n}(B) \rightarrow 0 .
$$

Due to the short exact sequence

$$
\begin{aligned}
0 & \rightarrow R / m \\
(\overline{1} & \rightarrow \bar{p})
\end{aligned}
$$

we obtain $\Omega_{R / V} \otimes R / m \neq 0$ and thus $\Omega_{R / V}$ is faithfully flat over $R$. Since $\mathrm{H}_{m}^{n}(B) \neq 0, \Omega_{R / V} \otimes_{R} \mathrm{H}_{m}^{n}(B) \neq 0$. Hence from (18) it follows that $\mathrm{H}_{m}^{n-1}\left(\Omega_{B / R}\right) \neq 0$.

Conversely, suppose that $\mathrm{H}_{m}^{n-1}\left(\Omega_{B / R}\right) \neq 0$. Consider the short exact sequence obtained via base change: $R \hookrightarrow B$ (spectral sequence)

$0 \rightarrow \operatorname{Ext}_{B}^{1}\left(\Omega_{B / R}, B^{*}\right) \rightarrow \operatorname{Ext}_{R}^{1}\left(\Omega_{B / R}, R\right) \rightarrow \operatorname{Hom}_{B}\left(\Omega_{B / R}, \operatorname{Ext}_{R}^{1}(B, R)\right)$.

Note that since $(R, m)$ is complete, given any $R$-module $M, \operatorname{Ext}_{R}^{i}(M, R)$ is $m$-separated - actually it is linearly compact for every $i \geq 0([9])$. This can be checked by considering a free resolution $F_{\bullet}$ of $M$ and then applying $\operatorname{Hom}_{R}\left(F_{\bullet}, R\right)$ and noticing that for any continuous $R$-linear map $f: T_{1} \rightarrow T_{2}$ between two linearly compact $m$-separated $R$-modules $\operatorname{Im} f$ and Ker $f$ are both linearly compact, $m$-separated closed submodules of $T_{2}$ and $T_{1}$ respectively $([9])$.

Since $\operatorname{Ext}_{R}^{1}(B, R)$ is $m$-separated and $\Omega_{B / R}=p^{n} \Omega_{B / R}$ for every $n>$ 0 , the right end term of the above exact sequence is 0 . By local duality, $\mathrm{H}_{m}^{n-1}\left(\Omega_{B / R}\right) \neq 0$ implies $\operatorname{Ext}_{R}^{1}\left(\Omega_{B / R}, R\right) \neq 0$. Hence from the above exact sequence $\operatorname{Ext}_{B}^{1}\left(\Omega_{B / R}, B^{*}\right) \neq 0$ and thus $B^{*} \neq 0$.

If $\mathrm{H}_{m}^{n-1}\left(\Omega_{B / V}\right) \neq 0$, same argument as above shows that $\operatorname{Hom}_{R}(B, R)$ $\neq 0$.

Now let us deal with the ramified case. In this case the proof that $\operatorname{Ext}_{R}^{1}\left(\Omega_{B / R}, R\right) \neq 0$ implies $\operatorname{Hom}_{R}(B, R) \neq 0$ is same as above. Our proof for the reverse implication needs a modification due to the fact that in this case $V \rightarrow R$ is not geometrically regular and hence $\Omega_{R / V}$ is not necessarily flat. We have the following exact sequence:

$$
0 \rightarrow N \rightarrow \Omega_{R / V} \otimes_{R} B \stackrel{\phi}{\rightarrow} \Omega_{B / V} \rightarrow \Omega_{B / R} \rightarrow 0
$$


Since $R$ is ramified, $R=S[X] /(f(X))$, where $S=V\left[\left[X_{1}, \ldots, X_{n-1}\right]\right]$ and $f(X)$ is an Eisenstein polynomial in $S[X]$. Then $\Omega_{R / S}=R /\left(f^{\prime}\right)$. By lemma 2.2 and the JZ sequence we have the following short exact sequence:

$0 \rightarrow \Omega_{S / V} \otimes_{S} R \rightarrow \Omega_{R / V} \rightarrow R / f^{\prime} R \rightarrow 0$

Tensoring this sequence with $B$ we obtain the following exact sequence:

$$
0 \rightarrow \Omega_{S / V} \otimes_{S} B \rightarrow \Omega_{R / V} \otimes_{R} B \rightarrow B / f^{\prime} B \rightarrow 0 \ldots \ldots \ldots \ldots \ldots \text { (20) }
$$

Let $q$ denote the maximal ideal of $S$. Then for any $R$-module $M, H_{q}^{i}(M)=H_{m}^{i}(M)$ and $\operatorname{Hom}_{R}(B, R) \simeq \operatorname{Hom}_{S}(B, S)$. Since $\mathrm{H}_{m}^{n}(B) \neq 0$ and $\Omega_{S / V}$ is faithfully flat over $S$, it follows that $\mathrm{H}_{m}^{n}\left(\Omega_{S / V} \otimes B\right) \simeq \Omega_{S / V} \otimes \mathrm{H}_{m}^{n}(B) \neq 0$. We have $\left(\Omega_{S / V} \otimes \mathrm{H}_{m}^{n}(B)\right)^{\vee} \simeq$ $\operatorname{Hom}\left(\Omega_{S / V}, \mathrm{H}_{m}^{n}(B)^{\vee}\right) \simeq \operatorname{Hom}\left(\Omega_{S / V}, B^{*}\right) \simeq \bigoplus_{1}^{n-1} B^{*}$ (lemma in i) b) theorem 3.6). Hence from the above exact sequence we obtain $\mathrm{H}_{m}^{n}\left(\Omega_{R / V} \otimes\right.$ $B) \neq 0$.

We also obtain an exact sequence (lemma 2.4) via $V \rightarrow S \rightarrow B$ :

$0 \rightarrow \Omega_{S / V} \otimes_{S} B \rightarrow \Omega_{B / V} \rightarrow \Omega_{B / S} \rightarrow 0 \ldots \ldots \ldots \ldots \ldots \ldots \ldots$ (21)

In (19) let $W=\operatorname{Im} \phi$. From (19), (20) and (21) we get the following commutative diagram of exact sequences:

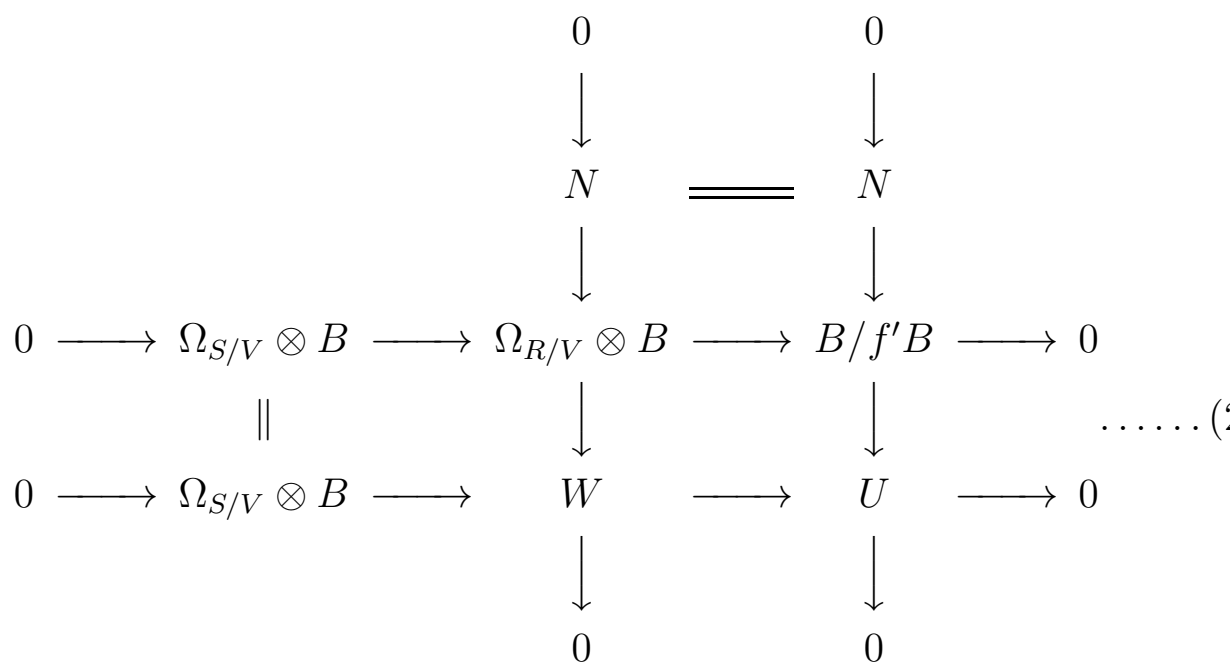

From the exact column on the right end we obtain $\mathrm{H}_{m}^{n}(N)=0$. Hence from the middle column we obtain $\mathrm{H}_{m}^{n}\left(\Omega_{R / V} \otimes B\right) \simeq \mathrm{H}_{m}^{n}(W) \neq 0$. Now applying local cohomology to the exact sequence (from 19):

$$
0 \rightarrow W \rightarrow \Omega_{B / V} \rightarrow \Omega_{B / R} \rightarrow 0
$$

We obtain our required result $\mathrm{H}_{m}^{n-1}\left(\Omega_{B / R}\right) \neq 0$ (by part i). 
When $\Omega_{S / V}$ is not flat over $S$, i.e. when $S=V, R=S[X] /(f(X))$ as mentioned earlier, $\operatorname{dim} R=1$ and $\operatorname{dim} B=1$. Then $\Omega_{B / V}$ is p-torsion and $H_{p}^{0}\left(\Omega_{B / V}\right)=\Omega_{B / V} \neq 0$.

Corollaries. With $R, B$ as in the above theorem we have the following

i) $\mathrm{H}_{m}^{n-1}\left(\Omega_{B / R}\right) \neq 0$.

This follows due to André's proof ([2]) of the direct summand conjecture.

ii) $\operatorname{Ext}_{B}^{1}\left(\Omega_{B / R}, B^{*}\right) \neq 0$.

This follows from the proof of the theorem.

Remark. We do not know whether part ii) of the above theorem is valid in equicharacteristic zero.

4.2. Our next proposition points out a relation between local cohomologies of $B$ and that of $\Omega_{B / R}, \Omega_{B / V}$.

Proposition. Let $(R, m)$ be a complete unramified regular local ring of dimension $n$ with coefficient ring $V$ and let $B$ be its absolute integral closure.

Then, for every $i \geq 0$, there exists a short exact sequence:

$$
0 \rightarrow \mathrm{H}_{m}^{i}\left(\Omega_{B / V}\right) \rightarrow \mathrm{H}_{m}^{i}\left(\Omega_{B / R}\right) \rightarrow \Omega_{R / V} \otimes_{R} \mathrm{H}_{m}^{i+1}(B) \rightarrow 0
$$

Proof. Consider the exact sequence (lemma 2.4):

$$
0 \rightarrow \Omega_{R / V} \otimes_{R} B \rightarrow \Omega_{B / V} \rightarrow \Omega_{B / R} \rightarrow 0
$$

. Since $\Omega_{R / V}$ is $R$-flat and $\Omega_{R / V} \otimes_{R} R / m \neq 0, \Omega_{R / V}$ is faithfully flat as an $R$-module. Hence $\mathrm{H}_{m}^{i}\left(\Omega_{R / V} \otimes_{R} B\right)=\Omega_{R / V} \otimes_{R} \mathrm{H}_{m}^{i}(B)$ is non-null whenever $\mathrm{H}_{m}^{i}(B)$ is so.

Claim. For any $B$-module $M, \Omega_{B / V} \otimes_{B} \mathrm{H}_{m}^{i}(M)=0$. Same result holds for $\Omega_{B / R}$.

Proof of the Claim. $\operatorname{Hom}_{R}\left(\Omega_{B / V} \otimes_{B} \mathrm{H}_{m}^{i}(M), E\right) \simeq \operatorname{Hom}_{B}\left(\Omega_{B / V}\right.$, $\left.\operatorname{Hom}_{R}\left(\mathrm{H}_{m}^{i}(M), E\right)\right) \simeq \operatorname{Hom}_{B}\left(\Omega_{B / V}, \operatorname{Ext}_{R}^{n-i}(M, R)\right)$ (local duality). Since $\operatorname{Ext}_{R}^{n-i}(M, R)$ is Hausdorff in the $m$-adic topology and $\Omega_{B / V}=p^{n} \Omega_{B / V}$, for $n>0$, we have $\operatorname{Hom}_{B}\left(\Omega_{B / V}, \operatorname{Ext}_{R}^{n-i}(M, R)\right)=0$ and hence the claim follows.

Now the assertion follows from noticing that the map: $\mathrm{H}_{m}^{i}\left(\Omega_{R / V} \otimes_{R}\right.$ $B) \rightarrow \mathrm{H}_{m}^{i}\left(\Omega_{B / V}\right)$ is the composition of the following two maps:

$\Omega_{R / V} \otimes_{R} \mathrm{H}_{m}^{i}(B) \simeq \Omega_{R / V} \otimes_{R} B \otimes_{B} \mathrm{H}_{m}^{i}(B) \rightarrow \Omega_{B / V} \otimes_{B} \mathrm{H}_{m}^{i}(B)$ and $\Omega_{B / V} \otimes_{B} \mathrm{H}_{m}^{i}(B) \rightarrow \mathrm{H}_{m}^{i}\left(\Omega_{B / V}\right)$

When $\Omega_{R / V}$ is not $R$-flat, i.e. when $\operatorname{dim} R=1$ i.e. $R=V, \Omega_{R / V}=0$ and the asserted sequence becomes obvious. 
Corollary 1. With $R, B$ as above, $\mathrm{H}_{m}^{i+1}(B) \neq 0 \Rightarrow \mathrm{H}_{m}^{i}\left(\Omega_{B / R}\right) \neq 0$ for $i \geq 0$.

Corollary 2. With $R, B$ as above, for every $i \geq 0$ there exists a short exact sequence:

0 .

$$
0 \rightarrow \bigoplus_{1}^{n-1} \operatorname{Ext}_{R}^{n-i-1}(B, R) \rightarrow \operatorname{Ext}_{R}^{n-i}\left(\Omega_{B / R}, R\right) \rightarrow \operatorname{Ext}_{R}^{n-i}\left(\Omega_{B / V}, R\right) \rightarrow
$$

In particular

$$
0 \rightarrow \bigoplus_{1}^{n-1} B^{*} \rightarrow \operatorname{Ext}_{R}^{1}\left(\Omega_{B / R}, R\right) \rightarrow \operatorname{Ext}_{R}^{1}\left(\Omega_{B / V}, R\right) \rightarrow 0 \text { is exact. }
$$

Proof. It follows from the lemma in part b), i) theorem 3.6 that $\Omega_{R / V}$ is a faithfully flat $R$-module, $\hat{\Omega}_{R / V} \simeq R^{n-1}$ and the sequence

$$
0 \rightarrow \cap_{t \geq 0}^{t} \Omega_{R / V} \rightarrow \Omega_{R / V} \rightarrow \hat{\Omega}_{R / V} \rightarrow 0
$$

is split exact. Since $R$ is complete, $\operatorname{Ext}_{R}^{n-i-1}(B, R)$ is m-separated (a proof of this fact is sketched in the proof of part ii), theorem 4.1). We have $\left(\Omega_{R / V} \otimes \mathrm{H}_{m}^{i+1}(B)\right)^{\vee} \simeq \operatorname{Hom}_{R}\left(\Omega_{R / V}, \mathrm{H}_{m}^{i+1}(B)^{\vee}\right) \simeq \operatorname{Hom}_{R}\left(\Omega_{R / V}\right.$, $\left.\operatorname{Ext}_{R}^{n-i-1}(B, R)\right)$. Since $\operatorname{Ext}_{R}^{n-i-1}(B, R)$ is m-separated, it follows from the above lemma that $\operatorname{Hom}_{R}\left(\Omega_{R / V}, \operatorname{Ext}_{R}^{n-i-1}(B, R)\right) \simeq \operatorname{Hom}_{R}\left(\oplus_{1}^{n-1} R\right.$,

$\left.\operatorname{Ext}_{R}^{n-i-1}(B, R)\right) \simeq{\underset{1}{n}}_{1}^{n-1} \operatorname{Ext}_{R}^{n-i-1}(B, R)$. Now applying $\operatorname{Hom}_{R}(-, E)$ to our asserted exact sequence in the above proposition the corollary follows.

\section{Remarks.}

1. If $\operatorname{dim} R \geq 2$, then $\mathrm{H}_{m}^{0}\left(\Omega_{B / V}\right) \simeq \mathrm{H}_{m}^{0}\left(\Omega_{B / R}\right)$.

This follows due to the fact that if $\operatorname{dim} R \geq 2$, then $H_{m}^{i}(B)=0, i=$ 0,1 and $\Omega_{R / V}$ is $R$-flat.

2. Part i) of theorem 4.1 (except $\mathrm{H}_{m}^{n}\left(\Omega_{B / R}\right)=0$ ) and results in 4.2 are not necessarily valid in equicharacteristic zero.

This follows mainly due to theorem 3.5.

4.3. Our final theorem deals with local cohomology of module of differentials with respect to the ideal $\left(x_{1}, \ldots, x_{n-1}\right)$ generated by a part of a regular system of parameters.

Theorem. Let $(R, m)$ be a complete regular local ring of dimension $n$ in mixed characteristics $p$ with co-efficient $\operatorname{ring} V$ and let $B$ be its absolute integral closure. Let $p, x_{1}, \ldots, x_{n-1}$ be a system of parameters of $R$ such that $x_{1}, \ldots, x_{n-1}$ form a regular system of parameters. Let $\underline{x}$ denote the ideal $\left(x_{1}, \ldots, x_{n-1}\right)$ in $R$. Then $\mathrm{H}_{\underline{x}}^{n-1}\left(\Omega_{B / V}\right) \neq 0$. 
Non-vanishing of $\mathrm{H}_{\underline{x}}^{n-1}\left(\Omega_{B / R}\right)$ implies the direct summand property for integral extensions of $R$.

Proof. For any $x \neq 0 \in R$ and for any $R$-module $M$, let $\tilde{K}(x ; M)$ denote the complex: $0 \rightarrow M \rightarrow M[1 / x] \rightarrow 0$ and $\tilde{K}\left(x_{1}, \ldots, x_{n-1} ; R\right)$ denote the complex $\underset{1}{\otimes} \tilde{K}\left(x_{i} ; R\right)$. The local cohomology modules of $\Omega_{B / V}$ with respect to $m$ are obtained by taking cohomologies of $\tilde{K}\left(x_{1}, \ldots, x_{n-1} ; R\right) \otimes \tilde{K}\left(p ; \Omega_{B / V}\right)$. Since this complex is a tensor product of two complexes, we obtain the following exact sequence:

$$
\mathrm{H}_{m}^{n-1}\left(\Omega_{B / V}\right) \rightarrow \mathrm{H}_{\underline{x}}^{n-1}\left(\Omega_{B / V}\right) \stackrel{\eta}{\rightarrow} \mathrm{H}_{\underline{x}}^{n-1}\left(\Omega_{B / V}[1 / p]\right) \rightarrow \mathrm{H}_{m}^{n}\left(\Omega_{B / V}\right) \rightarrow
$$
0 .

By theorem $4.1 \mathrm{H}_{m}^{n}\left(\Omega_{B / V}\right)=0$; hence $\eta$ is onto.

Let $q$ denote the prime ideal $\underline{x} \subset R$, then $q R_{q}$ is a maximal ideal in $R_{q}$. We have:

$\mathrm{H}_{\underline{x}}^{n-1}\left(\Omega_{B / V}[1 / p]\right)=\Omega_{B / V}[1 / p] \underset{R}{\otimes} \mathrm{H}_{\underline{x}}^{n-1}(R) \simeq \Omega_{B / V} \underset{R}{\otimes} \mathrm{H}_{\underline{x}}^{n-1}(R[1 / p]) \simeq$ $\Omega_{B / V} \otimes_{R} \mathrm{H}_{q R_{q}}^{n-1}\left(R_{q}\right) \simeq \Omega_{B / V} \otimes_{R} \mathrm{H}_{q \widehat{R}_{q}}^{n-1}\left(\widehat{R}_{q}\right) \simeq \mathrm{H}_{q \widehat{R}_{q}}^{n-1}\left(\Omega_{B_{q} / k} \otimes \widehat{R}_{q}\right), \quad(k=$ field of fractions of $V)$. By theorem 3.5 we have $\operatorname{Hom}_{R_{q}}\left(\Omega_{B_{q / k}}, R_{q}\right) \neq 0 \Rightarrow$ $\operatorname{Hom}_{R_{q}}\left(\Omega_{B_{q / k}}, \widehat{R}_{q}\right) \neq 0 \Rightarrow \operatorname{Hom}_{\widehat{R}_{q}}\left(\Omega_{B_{q / k}} \otimes \widehat{R}_{q}, \widehat{R}_{q}\right) \neq 0$. Hence, by local duality, it follows from above that $\mathrm{H}_{\underline{x}}^{n-1}\left(\Omega_{B / V}[1 / p]\right) \neq 0$.

Since $\eta$ is onto in (23) it follows that $\mathrm{H}_{\underline{x}}^{n-1}\left(\Omega_{B / V}\right) \neq 0$.

Now let us assume $\mathrm{H}_{\underline{x}}^{n-1}\left(\Omega_{B / R}\right) \neq 0$. By similar arguments as for exactness in (23) we obtain the following exact sequence

$$
\mathrm{H}_{m}^{n-1}\left(\Omega_{B / R}\right) \rightarrow \mathrm{H}_{\underline{x}}^{n-1}\left(\Omega_{B / R}\right) \rightarrow \mathrm{H}_{\underline{x}}^{n-1}\left(\Omega_{B / R}[1 / p]\right) \rightarrow \mathrm{H}_{m}^{n}\left(\Omega_{B / R}\right) \rightarrow
$$
0 ........

By arguments as above we have $\mathrm{H}_{\underline{x}}^{n-1}\left(\Omega_{B / R}[1 / p]\right)=\mathrm{H}_{q R q}^{n-1}\left(\Omega_{B_{q} / R_{q}}\right)=$ 0 (corollary to lemma 2.1) and hence $\mathrm{H}_{\underline{x}}^{n-1}\left(\Omega_{B / R}[1 / p]\right)=0$. It follows from (24) that the sequence $\mathrm{H}_{m}^{n-1}\left(\Omega_{B / R}\right) \rightarrow \mathrm{H}_{\underline{x}}^{n-1}\left(\Omega_{B / R}\right) \rightarrow 0$ is exact. Hence the assertion follows by part ii) theorem 4.1 .

Corollary. If $\mathrm{H}_{p}^{0}\left(\mathrm{H}_{\underline{x}}^{n-1}\left(\Omega_{B / V}\right)\right) \neq 0$, then $\mathrm{H}_{m}^{n-1}\left(\Omega_{B / V}\right) \neq 0$.

Suppose $\mathrm{H}_{p}^{0}\left(\mathrm{H}_{\underline{x}}^{n-1}\left(\Omega_{B / V}\right)\right) \neq 0$. Then, in the exact sequence (23), $\eta$ is not an isomorphism and hence $\mathrm{H}_{m}^{n-1}\left(\Omega_{B / V}\right) \neq 0$.

Remark. With notations as above if $I$ is an ideal in $R$ generated by a system of parameters of length $n-1$ such that $p \in I$, then $\mathrm{H}_{I}^{n-1}\left(\Omega_{B / R}\right)=0$ and $\mathrm{H}_{I}^{n-1}\left(\Omega_{B / V}\right)=0$.

This has been pointed out in part i) of theorem 4.1.

4.4. We here mention a couple of questions that came up during this study and whose answers we do not know at present. 
1. Let $(R, m)$ be a complete regular local ring of dimension $n$ of equicharacteristic 0 and let $B$ be its absolute integral closure. Is $\mathrm{H}_{m}^{n-1}\left(\Omega_{B / R}\right) \neq 0$ ?

2. Let $(R, m)$ be a complete regular local ring of dimension $n$ of mixed characteristic $p$ with coefficient ring $V$ and let $B$ be its absolute integral closure. Let $p, x_{1}, \ldots, x_{n-1}$ be a system of parameters of $R$ such that $x_{1}, \ldots, x_{n-1}$ form a part of a regular system of parameters.

a) Is $\mathrm{H}_{\underline{x}}^{n-1}\left(\Omega_{B / R}\right) \neq 0$ ?

b) $\mathrm{Is}_{p}^{0}\left(\mathrm{H}_{\underline{x}}^{n-1}\left(\Omega_{B / V}\right)\right) \neq 0$ ?

Remark. It can be shown that an affirmative answer for b) implies an affirmative answer for a).

\section{REFERENCES}

[1] M. André, Homologies des algébres commutative, Springer Verlag Berlin Heidelgerg New York (1974).

[2] Y. André, La conjecture de facteur direct, Publ. Math. IHES 127, 71-93 (2018).

[3] B. Bhatt, On the direct summand conjecture and its derived variant, Invent. Math. 212.2(2018), 297-317.

[4] O. Gabber \& L. Ramero, Foundations For Alomost Ring Theorey, arXiv:math/0409584V7[Math AG] Feb 2012.

[5] A. Grothendieck \& J. Dieudonné, EGA IV, Publ. Math. IHES 20 (1964).

[6] R. Hartshorne, Local Cohomology, Lecture Notes in Math, 41 (1967), SpringerVerlag. Berlin. Heidelberg. NewYork.

[7] M. Hochster, Contracted ideals from integral extensions of regular rings, Nagoya Math. J. 51(1973), 25-43.

[8] M. Hochster, Canonical elements in local cohomology modules and the direct summand conjecture, J. of Algebra, vol. 84, No. 3, October (1983), 503-553.

[9] I. G. Macdonald, Duality over complete local rings, Topology, vol. 1, 213-235, 1962.

[10] M. Nagata, Local Rings, R. E. K. Publ. Company, Huntington, New York, 1975.

[11] D. Popescu, General Nèron desingularization and approximation, Nagoya Math. J., 104 (1986), 85-115.

[12] M. Raynaud et L. Gruson, Critères de platitude et projectivité, Inventiones Math., 13, (1971), 1-89.

[13] J. P. Serre, Local Fields, Grad. text in Math., Springer Verlag New York Heidelberg Berlin.

[14] R. G. Swan, Nèron-Popescu desingularization, Proc. Alg. and Geom (1995), 135-192.

[15] O. Zariski, On the purity of the branch locus of algebraic functions, Proc. Nat. Acad. U. S. 44(1958), 791-796.

Department of Mathematics, University of Illinois at Urbana-Champaign, 1409 West Green Street, Urbana, Illinois 61801 